\title{
Expression and predictive value of HIF-1 $\alpha$ and VEGF in patients with burns following treatment
}

\author{
ZHUFENG LIU ${ }^{1}$, LING LIU ${ }^{1}$, XUEJUAN CHENG $^{2}$ and LIMING GAO ${ }^{1}$ \\ ${ }^{1}$ Department of Burn Dermatology, Jinan City People's Hospital, Jinan, Shandong 271100; \\ ${ }^{2}$ Department of Nursing, The People's Hospital of Zouping City, Binzhou, Shandong 256200, P.R. China
}

Received January 29, 2020; Accepted May 15, 2020

DOI: $10.3892 / \mathrm{etm} .2020 .9270$

\begin{abstract}
The present study aimed to investigate the expression and predictive value of serum hypoxia-inducible factor- $1 \alpha(\mathrm{HIF}-1 \alpha)$ and vascular endothelial growth factor (VEGF) in patients with burns following treatment. A total of 84 patients with burns treated in Jinan City People's Hospital (Jinan, China) between June 2015 and August 2017 were selected and their clinical information was collected. The expression levels of HIF-1 $\alpha$ and VEGF before and after treatment were detected via ELISA, and HIF-1 $\alpha$ and VEGF levels in patients with effective and ineffective treatment were compared. The predictive values of HIF-1 $\alpha$ and VEGF in clinical efficacy were determined using receiver operating characteristic (ROC) curves, and independent risk factors affecting treatment inefficacy were analyzed via multivariate logistic regression. It was revealed that HIF-1 $\alpha$ decreased significantly $(\mathrm{P}<0.05)$ while VEGF significantly increased in patients after treatment. Patients with effective treatment presented significantly lower HIF-1 $\alpha$ levels and higher VEGF levels compared with those with ineffective treatment. The ROC curve indicated that the area under the curve (AUC) of HIF-1 $\alpha$ for treatment efficacy was 0.795 , the $95 \%$ CI was 0.666-0.924, the specificity and sensitivity were 68.75 and $80.88 \%$, respectively, and the Youden index was $49.63 \%$. For VEGF, the AUC, 95\% CI, specificity, sensitivity and Youden index were 0.826 , $0.725-0.928,68.75,82.35$ and $51.10 \%$ respectively. Moreover, under the joint detection of HIF- $1 \alpha$ and VEGF, the AUC was $0.847,95 \%$ CI was $0.746-0.947$, specificity and sensitivity were 87.50 and $66.18 \%$, respectively, with a Youden index of $53.68 \%$. Multivariate analysis demonstrated that higher HIF-1 $\alpha$ level, lower VEGF level and higher burn degree before treatment were independent risk factors for treatment inefficacy. HIF-1 $\alpha$ levels decreased and VEGF levels increased in burn patients
\end{abstract}

Correspondence to: Dr Liming Gao, Department of Burn Dermatology, Jinan City People's Hospital, 1 Xuehu Street, Changshao North Road, Laiwu, Jinan, Shandong 271100, P.R. China E-mail: qweglm@163.com

Key words: hypoxia-inducible factor-1 $\alpha$, vascular endothelial growth factor, burn, efficacy prediction after treatment. HIF-1 $\alpha$ and VEGF before treatment may therefore serve as predictors for treatment efficacy.

\section{Introduction}

Burns are the most common form of soft tissue injury and can result in extensive wound injuries while increasing the risk of infection, systemic inflammation and sepsis in patients; moreover, the incidence of severe infection complications after burns increases mortality by $40 \%(1,2)$. Generally, patients will enter into a high metabolic state following a burn, with an accelerated metabolic rate; such injury lasting for several years will result in massive lean muscle loss, immune damage and delayed wound healing (3). The treatment objective in burn patients is to prevent infection and optimize recovery function (4). Although deep burn wounds are be removed as soon as possible and local antibiotics and dressings are used in time, the treatment methods of patients vary due to their different clinical characteristics. In addition, the needs of patients with burn are specific, while contrary to this, early treatment is often empirical, which delays effective treatment. Furthermore, certain patients develop resistance to treatment, leading to decreased efficacy (5). Therefore, better indicators are urgently needed to fill the gap of lack of biological indicators to predict efficacy (6).

Serum hypoxia-inducible factor- $1 \alpha(\mathrm{HIF}-1 \alpha)$, which plays a role in the process of angiogenesis and healing in patients, changes with the cell's perception of oxygen, and its level reflects the cell's oxygen content (7-9). HIF-1 $\alpha$ level is often higher than normal under hypoxic conditions, and when it rises, it further activates vascular endothelial growth factor (VEGF) $(10,11)$. VEGF is a more specific angiogenic factor that promotes the growth of vascular endothelial cells, which can not only drive but also promote angiogenesis (12). It is also associated with metastasis and angiogenesis of numerous tumors, and therefore can be used as a predictive indicator for certain tumors $(13,14)$. For example, Basagiannis et al (15) showed that VEGF induced VEGFR2 internalization through macrophage phagocytosis, which led to the activation of the neovascularization signaling pathway driven by VEGFR2 and angiogenesis. In addition, studies have shown that VEGF binds to the VEGF receptor on the endothelial cell membrane, causing autophosphorylation of the receptor, which in turn activates MAPK and realizing the mitogen characteristics of VEGF, thereby inducing endothelial cell proliferation $(16,17)$. However, the expression levels and 
predictive value of HIF-1 $\alpha$ and VEGF in patients with burns after treatment remain poorly understood at present.

Therefore, the present study detected the expression levels of HIF- $1 \alpha$ and VEGF in burn patients after treatment, and observed their predictive value of curative effect, so as to provide the basis and direction for clinical practice.

\section{Materials and methods}

Patients. In total, 84 patients with burns, treated in Jinan City People's Hospital (Jinan, China) between June 2015 and August 2017, were selected as the study participants, including 48 males and 36 females, with an average age of $48.3 \pm 9.5$ years. The present study was approved by the Ethics Committee of Jinan City People's Hospital (Jinan, China) and all patients provided signed informed consent.

Inclusion and exclusion criteria. The inclusion criteria were as follows: i) All participants presented with mild or moderate burns for the first time, without prior amputation; ii) participants were willing to cooperate with the treatment and follow-up; iii) patients had complete clinical data; and iv) patients had a life expectancy of $>3$ months.

The exclusion criteria were patients with: i) Tumors; ii) acute infectious disease; iii) severe burns iv) liver or kidney dysfunction; v) complications associated with sepsis; vi) other severe inflammation; or vii) diabetes.

Reagents and instruments. HIF-1 $\alpha$ ELISA detection kit (cat. no. E-EL-H6066) and VEGF ELISA assay kit (cat. no. E-EL-H1601c) were purchased from Elabscience Biotechnology Co., Ltd. Moisturizing Burn Cream was obtained from Mebo Pharmaceutical Co., Ltd.

Treatment efficacy determination. Wound healing rates of $>95 \%$ was considered as wound healing. Ineffective treatment efficacy was defined by poor growth of granulation tissue on the wound surface, with a wound healing area of $<50 \%$. If the wound healing rate was $\geq 50 \%$, the therapeutic efficacy was considered to be effective. Wound healing rate $(\%)=($ total wound area before treatment-total wound area after treatment)/total wound area before treatment x $100 \%$.

Treatment methods. After the wound was cleaned with Iodophor mixed with $0.9 \%$ saline (1:5), the moisture exposed burn ointment (MEBO) was soaked in sterile gauze and applied on the wound evenly, and the outer layer was wrapped with medical gauze to ensure full drainage of the wound exudate. During initial stages of exudate, the dressing was changed twice a day, and decreased to once a day when the wound was clean. Patients were treated continuously for 21 days. During the treatment, the wound was kept clean to prevent infection, and the dressing was changed in time if there was any abnormality such as the red, swollen or unclean wound.

Sample collection and ELISA test. Aseptic venous blood $(5 \mathrm{ml})$ was collected from patients at 7 a.m. the next day after admission and at 7 a.m. the first day following the 21-day treatment regime and placed in a coagulant tube. Subsequently, the samples were immediately centrifuged at $3,000 \mathrm{xg}$ at $4^{\circ} \mathrm{C}$ for
Table I. Clinical data of patients $(n=84)$.

\begin{tabular}{lc}
\hline Characteristic & Value \\
\hline Sex, $\mathrm{n}(\%)$ & \\
Male & $48(57.14)$ \\
Female & $36(42.86)$ \\
Age (years), mean \pm standard deviation & $48.3 \pm 9.5$ \\
BMI (kg/m $\left.{ }^{2}\right)$, mean \pm standard deviation & $21.23 \pm 2.14$ \\
Burn degree, $\mathrm{n}(\%)$ & \\
Mild & $57(67.86)$ \\
Moderate & $27(32.14)$ \\
Wound area, $\mathrm{n}(\%)$ & \\
$\leq 15 \%$ & $66(78.57)$ \\
$>15 \%$ & $18(21.43)$ \\
Residence, $\mathrm{n}(\%)$ & \\
Urban & $72(85.71)$ \\
Rural & $12(14.29)$ \\
Treatment efficacy, $\mathrm{n}(\%)$ & \\
Effective & $68(80.95)$ \\
Ineffective & $16(19.05)$ \\
Smoking history, $\mathrm{n}(\%)$ & \\
Yes & \\
No & $21(25.00)$ \\
Alcoholism history, $\mathrm{n}(\%)$ & $63(75.00)$ \\
Yes & \\
No & $17(20.23)$ \\
Type of burn, $\mathrm{n}(\%)$ & \\
Thermal burn & \\
Electrical burn & \\
Chemical burn & \\
\hline
\end{tabular}

$10 \mathrm{~min}$ to separate the serum and then stored in the refrigerator at $-80^{\circ} \mathrm{C}$. ELISA kits (cat. nos. ab171577 and ab233625; Abcam), was employed to determine HIF-1 $\alpha$ and VEGF levels. The dilution concentrations of VEGF standard samples were $4,000,2,000,1,000,500,250,125,62.50$ and $0 \mathrm{pg} / \mathrm{ml}$, and the configuration concentrations of HIF-1 were 2,000, 1,000, 500, $250,125,62.5,31.25$ and $0 \mathrm{pg} / \mathrm{ml}$. Blank, standard and sample wells to be tested were set, in which $100 \mu 1$ sample diluent was added to the blank wells, $100 \mu \mathrm{l}$ standard substance was added to the standard wells and $100 \mu \mathrm{l}$ sample was added to the sample wells to be tested. The ELISA plate was coated and incubated at $37^{\circ} \mathrm{C}$ for $90 \mathrm{~min}$. After removing and shaking the liquid in the wells, $100 \mu$ l biotinylated antibody working solution was added into each well, and the ELISA plate was coated with VEGF and HIF-1 antibody and incubated at $37^{\circ} \mathrm{C}$ for $1 \mathrm{~h}$. Subsequently, the liquid in wells were removed and plates were washed three times. The liquid was pat dry and $100 \mu \mathrm{l}$ enzyme conjugate added to each well and incubated at $37^{\circ} \mathrm{C}$ for $30 \mathrm{~min}$ after coating. Following which, the solution was dried, and the plate was washed five times, followed by the addition of $90 \mu \mathrm{l}$ chromogenic reagent and a 15 -min incubation in the dark at $37^{\circ} \mathrm{C}$ after coating with enzyme binding buffer. Next, $50 \mu \mathrm{l}$ termination solution was added to each well, and 
Table II. Receiver operating characteristic data.

\begin{tabular}{lccccr}
\hline Parameter & $\begin{array}{c}\text { Area under } \\
\text { the curve }\end{array}$ & $95 \%$ CI & $\begin{array}{c}\text { Specificity } \\
(\%)\end{array}$ & $\begin{array}{c}\text { Sensitivity } \\
(\%)\end{array}$ & $\begin{array}{c}\text { Youden index } \\
(\%)\end{array}$ \\
\hline HIF-1 $\alpha$ & 0.795 & $0.666-0.924$ & 68.75 & 80.88 & 49.63 \\
VEGF & 0.826 & $0.725-0.928$ & 68.75 & 82.35 & 51.10 \\
Joint detection & 0.847 & $0.746-0.947$ & 87.50 & 66.18 & 53.68 \\
\hline
\end{tabular}

HIF, hypoxia-inducible factor; VEGF, vascular endothelial growth factor.
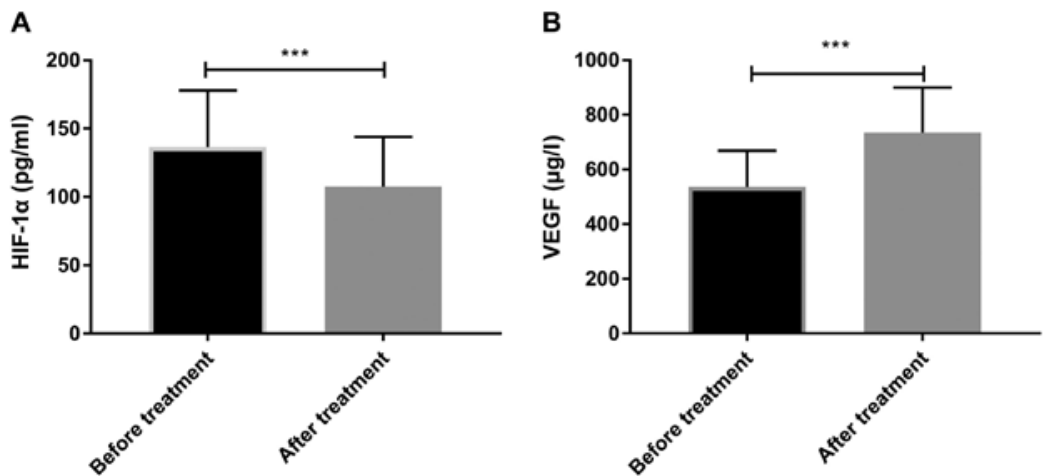

Figure 1. Changes in serum HIF-1 $\alpha$ and VEGF levels before and after treatment. (A) HIF-1 $\alpha$ decreased significantly following treatment $(\mathrm{t}=4.983$, $\mathrm{P}<0.001$ ). (B) VEGF significantly increased following treatment $(\mathrm{t}=8.826, \mathrm{P}<0.001) .{ }^{* * * *} \mathrm{P}<0.001$ vs. before treatment. HIF, hypoxia-inducible factor; VEGF, vascular endothelial growth factor.

the optical density value of each well was determined at a wavelength of $450 \mathrm{~nm}$ within $15 \mathrm{~min}$. The concentration was of HIF-1 $\alpha$ and VEGF in serum was then calculated.

Outcome measures. The HIF-1 $\alpha$ and VEGF levels were measured before and after treatment. All the patients were grouped according to the treatment efficacy after treatment: Patients with effective curative effects were included in the effective group, while patients with ineffective curative effects were included in the ineffective group, and their pre-treatment HIF-1 and VEGF levels were compared. In addition, the predictive value of HIF-1 $\alpha$ and VEGF in therapeutic efficacy was evaluated using an ROC curve, and the independent risk factors affecting treatment inefficacy were analyzed via multivariate logistic regression.

Statistical analysis. The data were statistical analyzed using SPSS 20.0 (IBM Corp.), and the required images were plotted using GraphPad Prism 7 (GraphPad Software, Inc.). The counting data represented by percentage $(\%)$ were compared using the $\chi^{2}$ test. The Kolmogorov-Smirnov test was employed to analyze the distribution of the data. The data were expressed as mean \pm standard deviation (SD). All the measurement data conformed to the normal distribution. Comparisons between the same group before and after treatment was performed using paired t-test, and those between two groups were performed using an independent sample Student's t-test, expressed as t. ROC curves were constructed to evaluate the predictive value of HIF-1 $\alpha$ and VEGF in terms of treatment efficacy. $\mathrm{P}<0.05$ indicated that there was a statistical difference between the two groups.

\section{Results}

Clinical data. The clinical data of patients were collected, including sex, age, BMI, burn degree, wound area, treatment efficacy, residence, smoking history and alcoholism history. (Table I).

Changes in HIF-1 $\alpha$ and VEGF before and after treatment. By observing the changes of HIF-1 $\alpha$ and VEGF before and after treatment in all patients, it was revealed that serum HIF-1 $\alpha$ levels (107.54 \pm 36.38$)$ were significantly lower following treatment compared with the levels before treatment $(136.36 \pm 41.54)(\mathrm{P}<0.05)$, while VEGF $(735.26 \pm 164.36)$ was significantly higher compared with before treatment (536.13 \pm 132.36$)(\mathrm{P}<0.05)$ (Fig. 1).

Predictive value of HIF-1 $\alpha$ and VEGF for treatment efficacy. The comparison of HIF-1 $\alpha$ and VEGF expression levels before treatment in patients with effective and ineffective treatment revealed that patients in the ineffective group had significantly higher HIF-1 $\alpha(\mathrm{P}<0.05)$, and significantly lower VEGF levels than those of patients in the effective group $(\mathrm{P}<0.05)$. The ROC curve exhibited that the AUC of HIF-1 $\alpha$ was 0.795 , and that of VEGF was 0.826, while the AUC of their joint detection was 0.847. (Fig. 2 and Table II)

Univariate analysis of treatment inefficacy in patients. The clinical data of patients in the effective group and the ineffective group were collected and analyzed via univariate analysis. It was revealed that there were no significant differences in sex, age, BMI, residence, smoking or alcoholism between the two 
Table III. Univariate analysis of treatment efficacy.

\begin{tabular}{|c|c|c|c|c|}
\hline Characteristic & Effective group $(\mathrm{n}=68)$ & Ineffective group $(\mathrm{n}=16)$ & $\mathrm{t} / \chi^{2}$-value & P-value \\
\hline Sex, n (\%) & & & 0.412 & 0.521 \\
\hline Male & $40(58.82)$ & $8(50.00)$ & & \\
\hline Female & $28(41.18)$ & $8(50.00)$ & & \\
\hline Age (years) & $47.8 \pm 8.6$ & $49.8 \pm 6.2$ & 0.876 & 0.383 \\
\hline BMI $\left(\mathrm{kg} / \mathrm{m}^{2}\right)$ & $21.28 \pm 1.65$ & $20.81 \pm 1.15$ & 1.007 & 2.285 \\
\hline Burn degree, n (\%) & & & 8.351 & 0.004 \\
\hline Mild & $51(75.00)$ & $6(37.50)$ & & \\
\hline Moderate & $17(25.00)$ & $10(62.50)$ & & \\
\hline Wound area, n (\%) & & & 5.849 & 0.016 \\
\hline$\leq 15 \%$ & $57(83.82)$ & $9(56.25)$ & & \\
\hline$>15 \%$ & $11(16.18)$ & $7(43.75)$ & & \\
\hline Residence, $\mathrm{n}(\%)$ & & & 0.322 & 0.571 \\
\hline Urban & $59(86.76)$ & $13(81.25)$ & & \\
\hline Rural & $9(13.24)$ & $3(18.75)$ & & \\
\hline Smoking history, $\mathrm{n}(\%)$ & & & 0.412 & 0.521 \\
\hline Yes & $16(23.53)$ & $5(31.25)$ & & \\
\hline No & $52(76.47)$ & $11(68.75)$ & & \\
\hline Alcoholism history, n (\%) & & & 0.278 & 0.598 \\
\hline Yes & $13(19.12)$ & $4(25.00)$ & & \\
\hline No & $55(80.88)$ & $12(75.00)$ & & \\
\hline Serum HIF- $1 \alpha$ level before treatment $(\mathrm{pg} / \mathrm{ml})$ & $141.56 \pm 34.33$ & $178.10 \pm 37.39$ & 3.767 & $<0.001$ \\
\hline Serum VEGF level before treatment $(\mu \mathrm{g} / \mathrm{l})$ & $555.17 \pm 124.76$ & $406.35 \pm 92.44$ & 4.482 & $<0.001$ \\
\hline
\end{tabular}

HIF, hypoxia-inducible factor; VEGF, vascular endothelial growth factor.
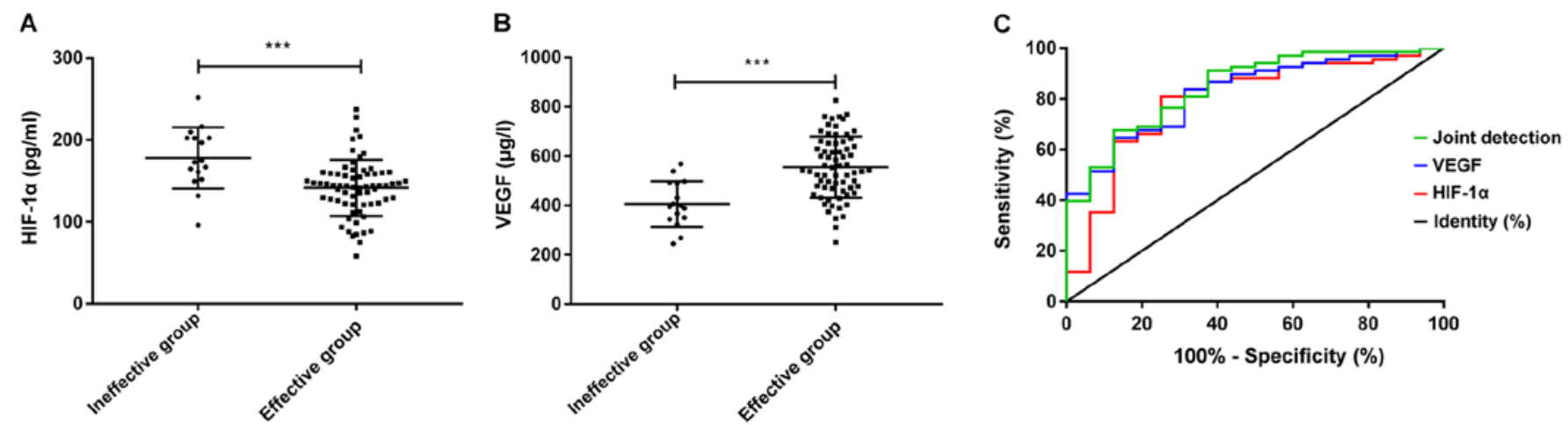

Figure 2. Predictive value of HIF-1 $\alpha$ and VEGF for treatment efficacy. (A) The level of HIF-1 $\alpha$ in the ineffective group was significantly higher compared with the effective group $(\mathrm{t}=3.767, \mathrm{P}<0.001)$. (B) VEGF levels in the ineffective group was significantly lower compared with the effective group $(\mathrm{t}=4.542$, $\mathrm{P}<0.001)$. ${ }^{* * * *} \mathrm{P}<0.001$. (C) The AUC of HIF-1 $\alpha$ for treatment efficacy was 0.795 , and when the cut-off point was 161.757 , its optimal specificity and sensitivity were 68.75 and $80.88 \%$, and the Youden index was $49.63 \%$. The AUC of VEGF for treatment efficacy was 0.826 , and when the cut-off point was 437.406, the optimal specificity and sensitivity were 68.75 and $82.35 \%$ respectively, and the Youden index was $51.10 \%$. While the AUC of the joint detection for treatment efficacy was 0.847 , and when the cut-off point was set as 0.847 , the optimal specificity and sensitivity were 87.50 and $66.18 \%$ and the Youden index was $53.68 \%$. HIF, hypoxia-inducible factor; VEGF, vascular endothelial growth factor; AUC, area under the curve.

groups; however, the burn degree, wound area, HIF-1 $\alpha$ level before treatment and VEGF level before treatment differed significantly between the groups $(\mathrm{P}<0.05)$ (Table III).

Multivariate analysis of treatment inefficacy. The indicators with significant differences in the univariate analysis were included in the assignment (see Table IV for the assignment table), and multivariate analysis was performed using the logistics regression equation. The results indicated that inefficacy of treatment was not associated with wound area, but was associated with burn degree [odds ratio (OR), 6.026; 95\% CI, 3.572-9.247], HIF-1 $\alpha$ level before treatment (OR, 3.475; 95\% CI, 1.386-6.834), and VEGF level before treatment (OR, 3.367; 95\% CI, 1.175-8.266) (Table V). 
Table IV. Assignment table.

Factors

Assignments

Burn degree

Wound area

Moderate $=1$, mild $=0$

HIF-1 $\alpha$ level before treatment

$>15 \%=1, \leq 15 \%=0$

VEGF level before treatment

Raw data analysis for continuous variables

Treatment efficacy

Raw data analysis for continuous variables Ineffective $=1$, effective $=0$

HIF, hypoxia-inducible factor; VEGF, vascular endothelial growth factor.

Table V. Multivariate analysis of survival.

\begin{tabular}{|c|c|c|c|c|c|c|c|}
\hline \multirow[b]{2}{*}{ Factors } & \multirow[b]{2}{*}{$\mathrm{B}$} & \multirow[b]{2}{*}{ SE } & \multirow[b]{2}{*}{ Wals } & \multirow[b]{2}{*}{ Sig. } & \multirow[b]{2}{*}{$\operatorname{Exp}(\mathrm{B})$} & \multicolumn{2}{|c|}{$\begin{array}{c}95 \% \text { CI for } \\
\operatorname{Exp}(B)\end{array}$} \\
\hline & & & & & & Lower & Upper \\
\hline Burn degree & 1.796 & 0.097 & 8.475 & 0.008 & 6.026 & 3.572 & 9.247 \\
\hline Serum HIF-1 $\alpha$ level before treatment & 1.237 & 0.026 & 5.649 & 0.004 & 3.475 & 1.386 & 6.834 \\
\hline Serum VEGF level before treatment $(\mu \mathrm{g} / \mathrm{l})$ & 1.245 & 0.118 & 5.287 & 0.003 & 3.367 & 1.175 & 8.266 \\
\hline
\end{tabular}

HIF, hypoxia-inducible factor; VEGF, vascular endothelial growth factor; B, regression coefficient; SE standard error; Wals, Wald Statistics; Sig., significance; Exp (B), odds ratio.

\section{Discussion}

Human skin serves immune and metabolic functions, while maintaining homeostasis in the human body, stabilizing body temperature and protecting the body from infection. Notably, when heat causes a large area of skin rupture, the physiological functions of the skin will change, increasing the risk of wound or systemic infection (18). HIF- $1 \alpha$ and VEGF are factors associated angiogenesis. Pagani et al (19) reported that HIF-1 $\alpha$ upregulation significantly enhanced tissue regeneration and promoted aging skin renewal and wound healing.

In the present study, the changes in serum HIF-1 $\alpha$ and VEGF levels were compared in patients before and after treatment. It was revealed that following treatment, the HIF-1 $\alpha$ level had significantly decreased, while the VEGF level increased. The reason behind the elevated expression of VEGF may be that the patients' skin was in a state of slow healing. However, in recent years, certain studies have also reported that the increase of VEGF is not beneficial to all burn patients. For instance, if the VEGF increases significantly after ocular alkali burn, the promotion of angiogenesis will result in the neovascularization of the cornea and damage the patient's vision, in which case anti-VEGF therapy should be implemented (20). While HIF-1 $\alpha$ is primarily and substantially expressed in skin wounds under anoxic conditions (21), and its decreased expression in the current study further suggested that the hypoxic state of the skin wound was further improved in the treatment process. Wound growth under hypoxic conditions may result in excessive growth of fibrous tissue and develop into scarring. Lei et al (22) reported that hypoxia-induced HIF-1 $\alpha$ expression significantly inhibited apoptosis and promoted cell proliferation in hypertrophic scar fibroblasts, but not in normal fibroblasts. Moreover, the overexpression of HIF-1 $\alpha$ can also cause endothelial barrier dysfunction, which may give rise to decreased vascular permeability and adversely affect patient recovery $(23,24)$. Therefore, treatments aim to reduce HIF-1 $\alpha$ levels and prevent the formation of hypertrophic scar after burns (25).

Subsequently, the pre-treatment expression levels of HIF- $1 \alpha$ and VEGF were compared between patients with effective and ineffective treatment, and it was revealed that the expression of HIF-1 $\alpha$ was significantly higher and VEGF was lower in the ineffective group compared with the effective group, suggesting that the levels of HIF-1 $\alpha$ and VEGF before treatment may be a predictor of patients' treatment efficacy. Therefore, the ROC curve was constructed to test their predictive value. It was revealed that the AUC of HIF- $1 \alpha$ for treatment efficacy was 0.795 , and the optimal specificity and sensitivity were 68.75 and $80.88 \%$ when the cut-off point was 161.757 , while the AUC of VEGF was 0.826 , and the optimal specificity and sensitivity were 68.75 and $82.35 \%$ when the cut-off point was 437.406, which also indicated that HIF-1 $\alpha$ and VEGF levels before treatment may predict the efficacy of treatment in patients. Moreover, differences were identified between the specificity and sensitivity of the two markers and, therefore, an assessment of the diagnostic value of measuring the levels of both markers was performed. The AUC of the joint detection was 0.847 , and the optimal specificity and sensitivity were 87.50 and $66.18 \%$, respectively, when the cut-off point was 0.847 , which was indicative that the differences were narrowed by joint detection. Subsequently, a multivariate 
analysis was performed based on the clinical data of patients with effective and ineffective treatment. This revealed a higher HIF-1 $\alpha$ level, lower VEGF level and higher burn degree were independent risk factors for treatment inefficacy.

However, there are also certain limitations to the present study. Primarily, the participants enrolled in this study were all burn patients without any healthy controls selected, which resulted in the poor understanding of the difference in indicators between the patients in this study and the normal population. Secondly, the present study excluded patients with severe burns. Compared with patients with mild or moderate burns, patients with severe burns are more prone to infection and shock. Therefore, it is hoped that patients with severe burns will be studied in the follow-up research to improve the conclusions of the present study. Thirdly, it has been reported in previous studies that cytokines produced as a result of hypoxic conditions in wounds, or inflammatory cells in excised wounds, can also regulate the activity of HIF; however, whether this has any impact on the present results remains unclear $(26,27)$. Finally, the current study did not further explore the mechanisms underlying the influence of HIF-1 $\alpha$ and VEGF on burn patients, which represents a target of future research.

Taken together, HIF-1 $\alpha$ level will decrease and VEGF expression will increase in burn patients after treatment, and HIF-1 $\alpha$ and VEGF levels before treatment may be of predictive value for treatment efficacy.

\section{Acknowledgements}

Not applicable.

\section{Funding}

No funding was received.

\section{Availability of data and materials}

The datasets used and/or analyzed during the current study are available from the corresponding author on reasonable request.

\section{Authors' contributions}

ZL conceived the study. LL and XC collected and analyzed data. LG helped perform statistical analysis. All authors read and approved the final manuscript.

\section{Ethics approval and consent to participate}

The current study was approved by the Ethics Committee of Jinan City People's Hospital, China. Patients who participated in this research, signed the informed consent and had complete clinical data.

\section{Patient consent for publication}

Not applicable.

\section{Competing interests}

The authors declare that they have no competing interests.

\section{References}

1. Rowan MP, Cancio LC, Elster EA, Burmeister DM, Rose LF, Natesan S, Chan RK, Christy RJ and Chung KK: Burn wound healing and treatment: Review and advancements. Crit Care 19: $243,2015$.

2. Kraft R, Herndon DN, Finnerty CC, Cox RA, Song J and Jeschke MG: Predictive value of IL-8 for sepsis and severe infections after burn injury - A clinical study. Shock 43: 222-227, 2015.

3. Clark A, Imran J, Madni T and Wolf SE: Nutrition and metabolism in burn patients. Burns Trauma 5: 11, 2017.

4. Oryan A, Alemzadeh E and Moshiri A: Burn wound healing: Present concepts, treatment strategies and future directions. J Wound Care 26: 5-19, 2017.

5. Hidalgo F, Mas D, Rubio M and Garcia-Hierro P: Infections in critically ill burn patients. Med Intensiva 40: 179-185, 2016.

6. Saaiq M, Ahmad S and Zaib MS: Burn wound infections and antibiotic susceptibility patterns at Pakistan institute of medical sciences, Islamabad, Pakistan. World J Plast Surg 4: 9-15, 2015.

7. Park SY, Lee SW, Kim HY, Lee WS, Hong KW and Kim CD: HMGB1 induces angiogenesis in rheumatoid arthritis via HIF-1a activation. Eur J Immunol 45: 1216-1227, 2015.

8. Kim Y, Nam HJ, Lee J, Park DY, Kim C, Yu YS, Kim D, Park SW, Bhin J, Hwang D, et al: Methylation-dependent regulation of HIF-1 $\alpha$ stability restricts retinal and tumour angiogenesis. Nat Commun 7: 10347, 2016.

9. MacLauchlan SC, Calabro NE, Huang Y, Krishna M, Bancroft T, Sharma T, Yu J, Sessa WC, Giordano F and Kyriakides TR: HIF- $1 \alpha$ represses the expression of the angiogenesis inhibitor thrombospondin-2. Matrix Biol 65: 45-58, 2018 .

10. Zhang X, Liu L, Wei X, Tan YS, Tong L, Chang R, Ghanamah MS, Reinblatt M, Marti GP, Harmon JW and Semenza GL: Impaired angiogenesis and mobilization of circulating angiogenic cells in HIF-1alpha heterozygous-null mice after burn wounding. Wound Repair Regen 18: 193-201, 2010.

11. Banyard DA, Adnani BO, Melkumyan S, Araniego CA and Widgerow AD: Endothelial progenitor cells and burn injury-exploring the relationship. Burns Trauma 4: 4, 2016.

12. Rumney RMH, Tozzi G, Kao A, Lanham SA, Kanczler JM, Kao AP, Thiagarajan L, Dixon JE, Tozzi G and Oreffo ROC: In vivo delivery of VEGF RNA and protein to increase osteogenesis and intraosseous angiogenesis. Sci Rep 9: 17745, 2019.

13. Feng Q, Zhang C, Lum D, Druso JE, Blank B, Wilson KF, Welm A, Antonyak MA and Cerione RA: A class of extracellular vesicles from breast cancer cells activates VEGF receptors and tumour angiogenesis. Nat Commun 8: 14450, 2017.

14. Morland C, Andersson KA, Haugen ØP, Hadzic A, Kleppa L, Gille A, Rinholm JE, Palibrk V, Diget EH, Kennedy LH, et al: Exercise induces cerebral VEGF and angiogenesis via the lactate receptor HCAR1. Nat Commun 8: 15557, 2017.

15. Basagiannis D, Zografou S, Murphy C, Fotsis T, Morbidelli L, Ziche M, Bleck C, Mercer J and Christoforidis S: VEGF induces signalling and angiogenesis by directing VEGFR2 internalisation through macropinocytosis. J Cell Sci 129: 4091-4104, 2016.

16. Grad S, Ertel W, Keel M, Infanger M, Vonderschmitt DJ and Maly FE: Strongly enhanced serum levels of vascular endothelial growth factor (VEGF) after poly-trauma and burn. Clin Chem Lab Med 36: 379-383, 1998.

17. Hwang C, Ucer S, Sorkin M, Loder S, Chung MT, Pagani C, Li J, Priest C, Breuler C, Vasquez K, et al: Identifying the role of and treatment targeting bone progenitor cell VEGF secretion on the niche supporting traumatic heterotopic ossification. Plast Reconstr Surg Glob Open 6: 21-22, 2018.

18. Church D, Elsayed S, Reid O, Winston B and Lindsay R: Burn wound infections. Clin Microbiol Rev 19: 403-434, 2006.

19. Pagani A, Aitzetmüller MM, Brett EA, König V, Wenny R, Thor D, Radtke C, Huemer GM, Machens HG and Duscher D: Skin rejuvenation through HIF-1 $\alpha$ modulation. Plast Reconstr Surg 141: 600e-607e, 2018.

20. Chen L, Zhong J, Li S, Li W, Wang B, Deng Y and Yuan J: The long-term effect of tacrolimus on alkali burn-induced corneal neovascularization and inflammation surpasses that of anti-vascular endothelial growth factor. Drug Des Devel Ther 12: 2959-2969, 2018.

21. Ciarlillo D, Celeste C, Carmeliet P, Boerboom D and Theoret C: A hypoxia response element in the Vegfa promoter is required for basal Vegfa expression in skin and for optimal granulation tissue formation during wound healing in mice. PLoS One 12: e0180586, 2017. 
22. Lei R, Li J, Liu F, Li W, Zhang S, Wang Y, Chu X and Xu J: HIF-1 $\alpha$ promotes the keloid development through the activation of TGF- $\beta /$ Smad and TLR4/MyD88/NF- $\kappa$ B pathways. Cell Cycle 18: 3239-3250, 2019.

23. Qi H, Wang P, Liu C, Li M, Wang S, Huang Y and Wang F: Involvement of HIF-1 $\alpha$ in MLCK-dependent endothelial barrier dysfunction in hypoxia. Cell Physiol Biochem 27: 251-262, 2011.

24. Tian KY, Liu XJ, Xu JD, Deng LJ and Wang G: Propofol inhibits burn injury-induced hyperpermeability through an apoptotic signal pathway in microvascular endothelial cells. Braz J Med Biol Res 48: 401-407, 2015.

25. Wu X, Li J, Yang X, Bai X, Shi J, Gao J, Li Y, Han S, Zhang Y, Han F, et al: miR-155 inhibits the formation of hypertrophic scar fibroblasts by targeting HIF-1 $\alpha$ via PI3K/AKT pathway. J Mol Histol 49: 377-387, 2018.
26. Albina JE and Reichner JS: Oxygen and the regulation of gene expression in wounds. Wound Repair Regen 11: 445-451, 2003.

27. Haroon ZA, Raleigh JA, Greenberg CS and Dewhirst MW: Early wound healing exhibits cytokine surge without evidence of hypoxia. Ann Surg 231: 137-147, 2000.

(†) $\odot$ This work is licensed under a Creative Commons Attribution-NonCommercial-NoDerivatives 4.0 International (CC BY-NC-ND 4.0) License. 